\title{
Evaluation of the utility of cardiac ultrasonography of patients in a surgical intensive care unit
}

\author{
Supparerk Prichayudha, Tatsana Uthaithammarat ${ }^{\mathrm{a}}$, Phornlert Chatrkaw ${ }^{\mathrm{b}}$, Sahadol Poonyathawon ${ }^{\mathrm{b}}$, Thammasak \\ Thawitsri ${ }^{\mathrm{b}}$, Kanya Kumwilaisak ${ }^{\mathrm{b}}$, Manasnun Kongwibulwut ${ }^{\mathrm{b}}$, Nalin Chokengarmwong \\ ${ }^{a}$ Department of Surgery, Chulalongkorn University, Bangkok 10330, Thailand \\ ${ }^{b}$ Department of Anesthesiology, Chulalongkorn University, Bangkok 10330, Thailand
}

\begin{abstract}
Background: Ultrasonography is being more commonly used by intensivists to assess the hemodynamic status of patients in intensive care units (ICUs) and for other purposes.

Objectives: To review the indications for and evaluate the impact of cardiac ultrasonography (CUS) on the management of patients in a surgical ICU (SICU).

Methods: We conducted a retrospective observational cohort study of patients in the SICU who underwent CUS performed by intensivists at King Chulalongkorn Memorial Hospital from January 2011 to March 2013. CUS was used to determine (1) preload (using inferior vena cava (IVC) diameter and collapsibility index), (2) cardiac contractility (using subjective assessment and fractional shortening), and (3) other miscellaneous findings.

Results: We included data from 157 patients (96 male and 61 female) whose age ranged from 15 to 99 years (mean 63.5 years) in the study. CUS was performed 190 times in these 157 patients. The most common indication for CUS was hemodynamic status assessment (78), followed by shock (69), oliguria (35), and other (8). CUS results led to 71 changes in management (37\% of cases); namely, fluid challenge (38), inotropic drug management (7), drainage of pleural/abdominal fluid (12), and other changes (14). A weakly-positive correlation between the IVC diameter and CVP (Pearson's $r=0.45$ ) was demonstrated. The overall mortality rate was $14.6 \%$.

Conclusions: CUS performed by intensivists can be used to assess the hemodynamic status of patients in the SICU, especially those with shock or oliguria, and lead to changes in the management of these patients.
\end{abstract}

Keywords: Cardiac ultrasonography, surgical ICU

Ultrasonography (US) has become an essential tool in almost every subdivision of medical practice including critical care medicine. Various applications of US have been used in ICUs, for instance, monitoring of hemodynamic parameters such as intravascular volume (preload) and cardiac contractility [1-8]. Despite the fact that US has some inherent limitations and is largely operator dependent, it enables clinicians to rapidly assess unstable patients by the bedside and is relatively inexpensive. Furthermore, US also aids in performing various bedside procedures (e.g., central venous catheter placement, and percutaneous drainage), and in detecting fluid collection, pneumothorax, and venous thrombosis. Hence, US has a significant impact in managing ICU patients and is replacing invasive hemodynamic monitoring in ICU [1-7]. Recently, several studies

Correspondence to: Supparerk Prichayudh, Department of Surgery, Chulalongkorn University, Bangkok 10330, Thailand. E-mail: supparerk.p@chula.ac.th evaluating the use of cardiac ultrasonography (CUS) in the surgical ICU (SICU) and critically-ill trauma patients have been reported [2-7]. However, these studies focused primarily on assessment of hemodynamic parameters (preload and contractility) and the majority was performed in a trauma population. The aim of the present study was to review the indications for and evaluate the impact of cardiac ultrasonography (CUS) results on the management of patients in the SICU.

\section{Methods}

After approval of the present observational study by our Institutional Review Board (approval No. 147/ 2015), we retrospectively reviewed the medical records of a cohort patients who underwent CUS in the SICU of King Chulalongkorn Memorial Hospital (a 1,400-bed university hospital in Bangkok, Thailand) from January 2011 to March 2013. The CUS was performed using an SSD-4000SV system (Hitachi Aloka Medical, Tokyo, Japan) by all the authors who 
are intensivists trained in the use of CUS. The main indications for CUS were (1) shock (a systolic blood pressure of $<90 \mathrm{mmHg}$, or presence of signs of poor tissue perfusion), (2) oliguria (urine output of $<0.5 \mathrm{~mL} / \mathrm{kg} / \mathrm{h}$ ), and (3) baseline assessment of hemodynamic status. The CUS was used mainly to determine (1) preload, (2) cardiac contractility, and (3) other miscellaneous findings.

Preload assessment was performed by measurement of the inferior vena cava (IVC) diameter, calculating the collapsibility index of IVC $\left(\mathrm{IVC}_{\mathrm{CI}}\right)$, or observing the ventricular wall kissing sign. The IVC diameter was measured using a subxiphoid view within $2 \mathrm{~cm}$ of the IVC-right atrial junction at the end of expiration in both mechanically ventilated and spontaneously breathing patients (Figure 1).
The $\mathrm{IVC}_{\mathrm{CI}}$ was calculated as followed:

$\mathrm{IVC}_{\mathrm{CI}}=$ maximum IVC diameter - minimum IVC diameter $\times 100 \%$

$$
\text { maximum IVC diameter }
$$

Any patients with IVC diameter less than $1.2 \mathrm{~cm}$, or $\mathrm{IVC}_{\mathrm{CI}}>50 \%$, or a positive ventricular wall kissing sign were classified as having hypovolemia [3, 9-11].

Cardiac contractility was assessed by (1) subjective assessment (observing overall contraction and grading as poor, normal, or hyperdynamic contraction), and (2) fractional shortening (FS) as shown in Figure 2.

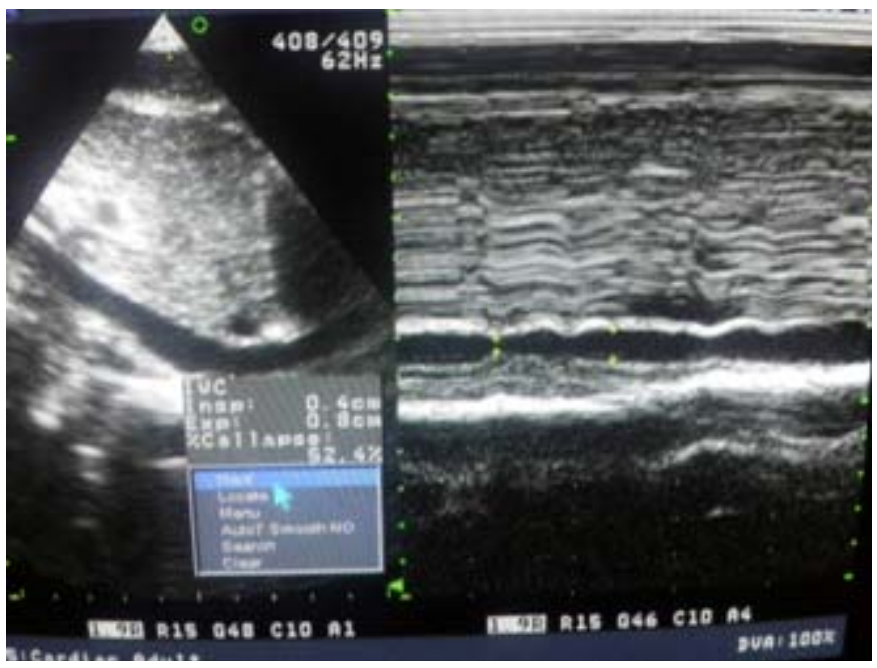

Figure 1. IVC diameter measurement via the subxiphoid view was performed within $2 \mathrm{~cm}$ of the IVC-right atrial junction at the end of expiration. The collapsibility index of IVC was also calculated.

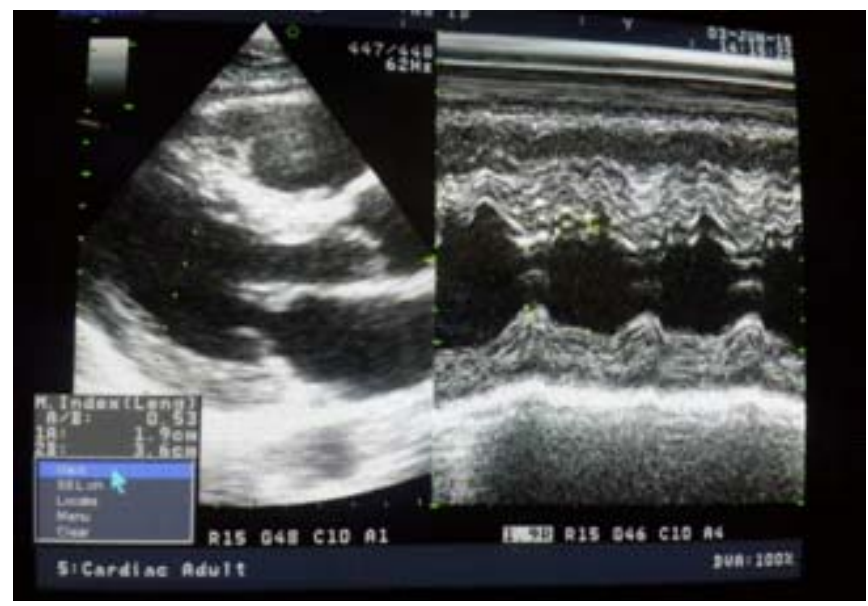

Figure 2. Fractional shortening was measured via the parasternal long axis view. 
According to the FS, the patient would be classified as having poor contraction when the FS was less than $25 \%[4,12]$. The other findings that were sought included the presence of pericardial fluid/ cardiac tamponade and gross valvular abnormalities.

Besides the hemodynamic assessment, the authors also performed additional US to detect miscellaneous problems, e.g. fluid collection, biliary tract problems, obstructive uropathy, and deep vein thrombosis (DVT) in patients suspected of having these particular problems. The main criterion for DVT diagnosis was the absence of venous compressibility. The adjunct criteria for DVT diagnosis included the presence of a thrombus, decrease of flow, and the absence of augmentation upon calf squeezing [13].

We classified the patients into 8 groups namely: general surgery, hepatobiliary-pancreatic surgery, vascular surgery, colorectal surgery, urology, trauma, orthopedics, and miscellaneous (e.g. plastic surgery, head and neck surgery). Data collected included demographic variables, patient groups, initial Sequential Organ Failure Assessment (SOFA) score, monitoring devices used, hemodynamic parameters, indications for CUS, and results of CUS. Outcomes including ICU days, ventilator days, and mortality were recorded. Change in management was defined as "alteration of a treatment plan because of the CUS results", and depended mainly on the clinical judgment of the intensivists.

Statistical analyses were performed using Microsoft Excel 2013, version 15.0.4420 (Microsoft Corp, Redmond, WA, USA). Descriptive ordinal data were analyzed and expressed as mean and standard deviation (SD). The linear association between the IVC diameter and central venous pressure (CVP) was analyzed using Pearson correlation coefficients and is represented by a scatter plot.

\section{Results}

From January 2011 to March 2013, CUS was performed 190 times in 157 patients (96 male and 61 female, mean age $63.5 \pm 19.4$ years) in the SICU. Their demographic data, monitoring device used, and inotropic drug use are shown in Table $\mathbf{1}$ and the indications of CUS are shown in Table 2.

Table 1. Demographic data, monitoring device, and inotropic drug use of 157 patients in the SICU who underwent cardiac ultrasonography

\begin{tabular}{ll}
\hline Sex & \\
$\quad$ Male & $96(61 \%)$ \\
$\quad$ Female & $61(39 \%)$ \\
Age (years, mean \pm SD) & $63.5 \pm 19.4$ \\
Average SOFA scores (mean \pm SD) & $6.1 \pm 4.4$ \\
Patient groups & \\
$\quad$ General surgery & $35(22 \%)$ \\
Trauma & $31(20 \%)$ \\
Vascular surgery & $28(18 \%)$ \\
Colorectal surgery & $25(16 \%)$ \\
$\quad$ Hepatobiliary-pancreatic surgery & $24(15 \%)$ \\
$\quad$ Urology & $7(4 \%)$ \\
$\quad$ Orthopaedics & $1(1 \%)$ \\
$\quad$ Miscellaneous & $6(4 \%)$ \\
Central venous pressure monitoring & $109(69 \%)$ \\
Arterial catheter & $133(85 \%)$ \\
Cardiac output monitoring & $12(8 \%)$ \\
$\quad$ Noninvasive & $9(6 \%)$ \\
Pulmonary arterial catheter & $3(2 \%)$ \\
Inotropic drugs & $74(47 \%)$
\end{tabular}

SOFA = Sequential Organ Failure Assessment Score, SD = standard deviation 
Table 2. Indications for cardiac ultrasonography

\begin{tabular}{llc}
\hline Indications & $\begin{array}{c}\text { No. of patients } \\
(\mathbf{n}=\mathbf{1 5 7})\end{array}$ & $\begin{array}{c}\text { Times cardiac ultrasonography } \\
\text { performed (n= 190) }\end{array}$ \\
\hline Shock & $58(37 \%)$ & $69(37 \%)$ \\
Oliguria & $28(18 \%)$ & $35(18 \%)$ \\
Assessment of hemodynamic status & $63(40 \%)$ & $78(41 \%)$ \\
Other: & $8(5 \%)$ & $8(4 \%)$ \\
$\quad$ Exclude pleural effusion & $3(2 \%)$ & $3(2 \%)$ \\
Exclude intraabdominal collection/ sepsis & $3(2 \%)$ & $3(2 \%)$ \\
$\quad$ Exclude pulmonary embolism & $2(1 \%)$ & $2(1 \%)$ \\
\hline
\end{tabular}

The CUS was performed on 137 patients (87\%) on a mechanical ventilator. The most common indication was baseline assessment of hemodynamic status (78 times), followed by shock (69 times), and oliguria (35 times).

CUS of 58 patients with shock was performed 69 times in the SICU to assess the hemodynamic status and causes of shock. According to our criteria of preload assessment, hypovolemia was identified 28 times, which led to fluid challenge 26 times following the clinical judgment of the intensivists. Poor left ventricular (LV) contraction was observed 10 times, which led to administration or increment of inotropic drugs on 5 occasions. One patient with global hypokinesia of the left ventricle was subsequently diagnosed with acute myocardial infarction. Valvular abnormalities were detected 6 times, all were subsequently confirmed by formal echocardiography conducted by cardiologists (2 aortic regurgitations, 1 aortic stenosis, 2 tricuspid regurgitations, and 1 mitral valve regurgitation). No cardiac tamponade detected in the present study.
CUS was performed 35 times in 28 patients with oliguria. Preload assessment revealed hypovolemia 15 times, which led to fluid challenge on 12 occasions. Poor LV contraction was identified 2 times, both instances treated by inotropic drug administration. In addition, urinary bladder obstruction was found 2 times, which led to urinary catheter changes.

Other miscellaneous findings from the additional US leading to changes in management were as follows: (1) pleural effusions leading to placement of percutaneous drainage (8 times), (2) intra-abdominal fluid/collection leading to placement of percutaneous drainage (4 times), (3) one intracardiac thrombus and 10 DVTs leading to administration of anticoagulant, and (4) cholecystitis leading to cholecystectomy (1 times). The US was also used to confirm the diagnosis of abdominal compartment syndrome by showing a narrow, compressed IVC in a patient with intra-abdominal hypertension who subsequently required decompressive laparotomy (Figure 3).

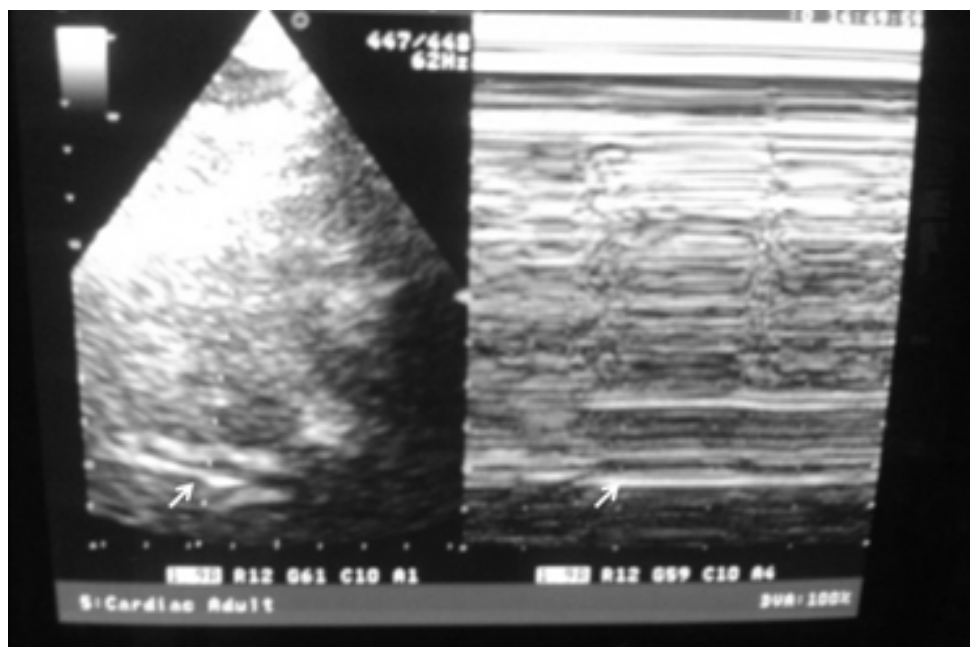

Figure 3. Ultrasonography of a 60 year-old male patient suffering abdominal compartment syndrome after a ventral hernia repair demonstrated a collapsed IVC (white arrow). The patient underwent decompressive laparotmy and survived. 
Overall, there were 71 changes in management dictated by 190 instances of CUS performed (38 fluid challenges, 7 inotropic drug managements, 11 anticoagulant administrations, and 15 other procedures). During the study period 23 patients died (15\% mortality). The outcomes of the patients are summarized in the Table 3.
An analysis of the correlation between CVP and IVC diameter was conducted for 109 patients who had a central venous catheter (98 mechanically ventilated and 11 spontaneous breathing). The correlation coefficient showed a weakly positive relationship between the IVC diameter (measured from CUS) and the CVP (Pearson's $r=0.45$ ) (Figure 4).

Table 3. Outcomes of 157 patients who underwent cardiac ultrasonography in the SICU

\begin{tabular}{ll}
\hline Ventilator days (mean \pm SD) & $10.9 \pm 14.8$ \\
Days in intensive care unit (mean \pm SD) & $12.9 \pm 14.7$ \\
Mortality (\%) & $23(15 \%)$ \\
$\quad$ Sepsis with multiorgan failure & 17 \\
Acute respiratory distress syndrome & 3 \\
Acute myocardial infarction & 1 \\
End stage heart disease & 1 \\
Severe hypoxic brain injury & 1 \\
Changes in management & 71 \\
Fluid challenges & 38 \\
Inotropic drug managements & 7 \\
Anticoagulant administrations & 11 \\
Urinary catheter changes & 2 \\
Drainages of pleural effusion & 8 \\
Drainages of intraabdominal fluid & 4 \\
Cholecystectomy & 1
\end{tabular}

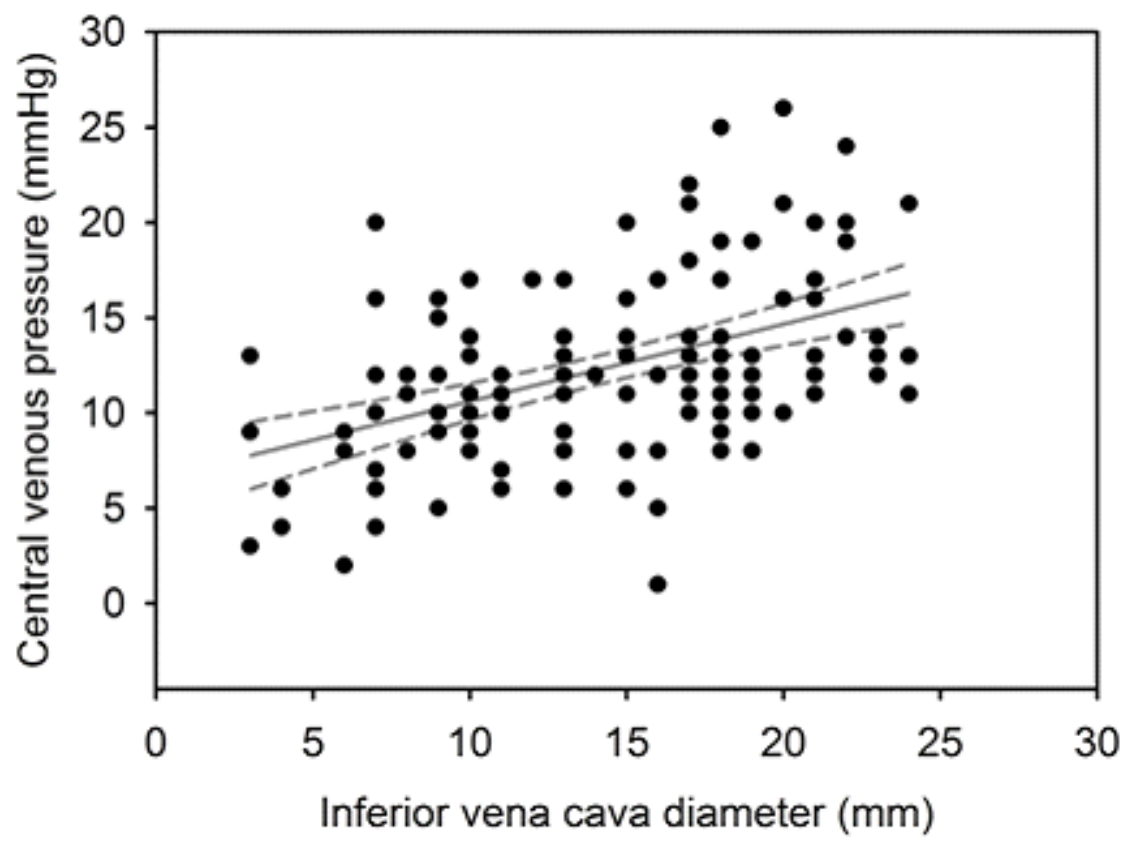

Figure 4. A weakly positive correlation was found between the diameter of the inferior vena cava and the central venous pressure, Pearson's $r=0.45$. The solid line shows the regression and dashed lines show the upper and lower $95 \%$ confidence intervals. 


\section{Discussion}

CUS performed by intensivists has played an important role in ICUs because it is a noninvasive test that provides useful hemodynamic information [18]. Furthermore, problem-focused US can help identify specific problems e.g., fluid collection, DVT, biliary tract diseases, and urinary tract obstruction, which are common in surgical patients as seen in the present study $[13,14]$. CUS can occasionally lead to changes in management in ICU patients $(18 \%-55 \%)[5,8]$. For instance, Ferrada et al. reported 55\% change in management in ICU patients with trauma who underwent CUS including volume management (79\%) and increase in inotropic drugs or vasopressors, or both (20\%) [5]. In the present study, most common change in management was fluid challenge, followed by invasive procedures, administration of anticoagulant, and inotropic drug management. However, these changes did not depend solely on the CUS findings because the decision to change the management plan in the present study depended mainly on the clinical judgment of the intensivists.

A correlation between IVC diameter and CVP or right atrial pressure (RAP) has been demonstrated by several studies including the present study [3, 9-11, 15, 16]. In the present study, we used end-expiratory IVC diameter to assess CVP because the diameter is not affected by inspiration, either in spontaneously breathing or mechanically-ventilated patients. We used $1.2 \mathrm{~cm}$ as a lower normal limit of IVC diameter because IVC diameter of $<1.2 \mathrm{~cm}$ is often seen with intravascular volume depletion and is specific for a low RAP $[9,10,15,16]$. The respiratory variation in IVC diameter has been used by other investigators to estimate RAP and to predict fluid responsiveness in ICU patients $[11,17,18]$. In the present study, we used the $\mathrm{IVC}_{\mathrm{CI}}$ of $>50 \%$ to define hypovolemia because this value correlates well with a low RAP and is usually associated with fluid responsiveness in patients with acute circulatory failure $[9,11]$.

The LV systolic function evaluation by CUS can be performed by subjective assessment and/or objective assessment. Volumetric measurement of the LV ejection fraction by Simpson's biplane method is a preferred over linear measurement [12]. Nevertheless, a prospective study comparing $3 \mathrm{LV}$ systolic function evaluation methods by CUS performed by intensivists showed that FS was more accurate than left ventricular outflow tract/velocity- time integral and Simpson's method in estimating cardiac index [3]. Moreover, noncardiologist physicians can measure FS relatively easily. We used FS as a component of cardiac contractility assessment in the present study. However, FS and cardiac output could not be correlated because the number of patients with cardiac output monitoring was low (7.6\%).

There are some limitations to the present study. First, this is a retrospective study with no clear management protocol in response to the CUS results because changes in management depended mainly on the judgment of the intensivists. Therefore, the amount of fluid administered when fluid challenge was performed or the type of inotropic drugs used were not uniform. Second, we did not have any data regarding response to treatment because there was no criterion to define response to treatment, especially in hemodynamic management (e.g., response to fluid challenge). Therefore, we could not identify the effectiveness of CUS-guided hemodynamic management.

In conclusion, we believe that CUS is a useful tool for evaluating patients in the SICU, especially those with acute hemodynamic problems (e.g., shock or oliguria) because it is a noninvasive test and is easy to perform. Along with clinical information, CUS and problem-focused US may help guide decision making in SICU patient management. Intensivists should be familiar with CUS technique so that it can be included in the ICU armamentarium.

\section{Conflict of interest statement}

The authors declare that there is no conflict of interest in this research.

\section{References}

1. Jensen MB, Sloth E, Larsen KM, Schmidt MB. Transthoracic echocardiography for cardiopulmonary monitoring in intensive care. Eur J Anaesthesiol. 2004; 21:700-7.

2. Manasia AR, Nagaraj HM, Kodali RB, Croft LB, Oropello JM, Kohli-Seth R, et al. Feasibility and potential clinical utility of goal-directed transthoracic echocardiography performed by noncardiologist intensivists using a small hand-carried device (SonoHeart) in critically ill patients. J Cardiothorac Vasc Anesth. 2005; 19:155-9.

3. Gunst M, Ghaemmaghami V, Sperry J, Robinson M, O’Keeffe T, Friese R, et al. Accuracy of cardiac function and volume status estimates using the 
bedside echocardiographic assessment in trauma/ critical care. J Trauma. 2008; 65:509-16.

4. Gunst M, Matsushima K, Sperry J, Ghaemmaghami V, Robinson M, O’Keeffe T, et al. Focused bedside echocardiography in the surgical intensive care unit: comparison of 3 methods to estimate cardiac index. J Intensive Care Med. 2011; 26:255-60.

5. Ferrada P, Murthi S, Anand RJ, Bochicchio GV, Scalea T. Transthoracic focused rapid echocardiographic examination: real-time evaluation of fluid status in critically ill trauma patients. J Trauma. 2011; 70:56-62.

6. Tchorz KM, Chandra MS, Markert RJ, Healy M, Anderson H, Ekeh P, et al. Comparison of hemodynamic measurements from invasive and noninvasive monitoring during early resuscitation. J Trauma Acute Care Surg. 2012; 72:852-60.

7. Murthi SB, Hess JR, Hess A, Stansbury LG, Scalea TM. Focused rapid echocardiographic evaluation versus vascular cather-based assessment of cardiac output and function in critically ill trauma patients. J Trauma Acute Care Surg. 2012; 72:1158-64.

8. Manno E, Navarra M, Faccio L, Motevallian M, Bertolaccini L, Mfochiv A, et al. Deep impact of ultrasound in the intensive care unit: the "ICU-sound" protocol. Anesthesiology. 2012; 117:801-9.

9. Kircher BJ, Himelman RB, Schiller NB. Noninvasive estimation of right atrial pressure from the inspiratory collapse of the inferior vena cava. Am J Cardiol. 1990; 66:493-6.

10. Ginghina C, Beladan CC, Iancu M, Calin A, Popescu BA. Respiratory maneuvers in echocardiography: A review of clinical applications. Cardiovasc Ultrasound. 2009; 7:42.

11. Muller L, Bobbia X, Toumi M, Louart G, Molinari N, Ragonnet B, et al. Respiratory variations of inferior vena cava diameter to predict fluid responsiveness in spontaneously breathing patients with acute circulatory failure: need for a cautious use. Crit Care.
2012; 16:R188

12. Lang RM, Bierig M, Devereux RB, Flachskampf FA, Foster E, Pellikka PA, et al. Recommendations for chamber quantification: a report from the American Society of Echocardiography's Guidelines and Standards Committee and the Chamber Quantification Writing Group, developed in conjunction with the European Association of Echocardiography, a branch of the European Society of Cardiology. J Am Soc Echocardiogr. 2005; 18:1440-63.

13. Prichayudh S, Tumkosit M, Sriussadaporn S, Samorn P, Pak-art R, Sriussadaporn S, et al. Incidence and associated factors of deep vein thrombosis in Thai surgical ICU patients without chemoprophylaxis: one year study. J Med Assoc Thai. 2015; 98:472-8.

14. Galvan DA, Matsushima K, Frankel HL. Ultrasound in the surgical intensive care unit. Isr Med Assoc J. 2011; 13:566-70.

15. Bendjelid K, Romand JA, Walder B, Suter PM, Fournier G. Correlation between measured inferior vena cava diameter and right atrial pressure depends on the echocardiographic method used in patients who are mechanically ventilated. J Am Soc Echocardiogr. 2002, 15:944-9.

16. Schefold JC, Storm C, Bercker S, Pschowski R, Oppert M, Kruger A, et al. Inferior vena cava diameter correlates with invasive hemodynamic measures in mechanically ventilated intensive care unit patients with sepsis. J Emerg Med. 2010; 38:632-7.

17. Feissel M, Michard F, Faller JP, Teboul JL. The respiratory variation in inferior vena cava diameter as a guide to fluid therapy. Intensive Care Med. 2004; 30: 1834-7.

18. Barbier C, Loubieres Y, Schmit C, Hayon J, Ricome JL, Jardin F, et al. Respiratory changes in inferior vena cava diameter are helpful in predicting fluid responsiveness in ventilated septic patients. Intensive Care Med. 2004; 30:1740-6. 